\title{
Reação de Cultivares de Feijoeiro Comum a Quatro Raças de Phaeoisariopsis griseola
}

\author{
Eder J. de Oliveira ${ }^{1,2}$, Ana L. Alzate-Marin',3, Aluízio Borém ${ }^{4}$, Carlos L. P. Melo' ${ }^{1}$, Everaldo G. \\ de Barros $^{1,5}$ \& Maurílio A. Moreira ${ }^{1,6}$
}

${ }^{1}$ Instituto de Biotecnologia Aplicada à Agropecuária - BIOAGRO/Universidade Federal de Viçosa, CEP 36570-000, Viçosa, MG, fax (031) 3899-2864; ${ }^{2}$ e-mail: eder@esalq.usp.br, ${ }^{3}$ e-mail:anaalzatem @ yahoo.com.br; ${ }^{4}$ Departamento de Fitotecnia, Universidade

Federal de Viçosa, e-mail: borem @ufv.br, ${ }^{5}$ Departamento de Biologia Geral, Universidade Federal de Viçosa, e-mail: ebarros@ufv.br, ${ }^{6}$ Departamento de Bioquímica e Biologia Molecular, Universidade Federal de Viçosa, e-mail: moreira@ufv.br

\section{(Aceito para publicação em 06/01/2004)}

Autor para correspondência: Maurílio A. Moreira

OLIVEIRA, E.J., ALZATE-MARIN, A.L., BOREM, A., MELO, C.L.P., BARROS, E.G. \& MOREIRA, M.A. Reação de cultivares de feijoeiro comum a quatro raças de Phaeoisariopsis griseola. Fitopatologia Brasileira 29:220-223. 2004.

\section{RESUMO}

A mancha-angular causada pelo fungo Phaeoisariopsis griseola, apresenta grande importância na cultura do feijoeiro (Phaseolus vulgaris) no Brasil. O desenvolvimento de cultivares resistentes tem sido proposto como maneira eficaz, eficiente e econômica para o controle da doença. Um dos primeiros passos no programa de melhoramento visando resistência à mancha-angular é a identificação e seleção de fontes de resistência. Neste contexto, este trabalho objetivou a caracterização de 58 cultivares de feijoeiro quanto a reação às raças $31.17,63.19,63.23$ e 63.55 de $P$. griseola. Os resultados mostraram que as cultivares Antioquia 8 e CAL 143, ambos de origem Andina, e Ecuador 299 e México 235, de origem Mesoamericana, apresentaram resistência às quatro raças testadas. As cultivares A 193 e Golden Gate 416 mostraram resistência a três das quatro raças testadas, podendo também, ser úteis em programas de melhoramento. Dentre as cultivares mais suscetíveis encontram-se as cultivares IPA 7419, AN 9022180, Bambuí, Compuesto Negro Chimaltengo, Guanajuato 10-A-5, Diamante Negro, Early Gallatin, Jamapa e Kentucky Wonder 780 e as cultivares de grãos tipo carioca AN 9022180, Aporé e Carioca 80. As novas fontes de resistência à mancha angular identificadas neste trabalho poderão ser utilizadas por programas de melhoramento do feijoeiro que visem a incorporação de genes de resistência de origem Andina ou Mesoamericana.

Palavras-chave adicionais: Phaseolus vulgaris, melhoramento genético vegetal, banco de germoplasma.

\section{ABSTRACT}

Reaction of common bean cultivars to four races of Phaeoisariopsis griseola

Angular leaf spot caused by the fungus Phaeoisariopsis griseola is a major disease of the common bean (Phaseolus vulgaris) in Brazil. The development of resistant cultivars has been proposed as an effective, efficient and economic way to control the disease. A breeding program aiming to develop cultivars resistant to angular leaf spot starts with the identification and selection of common bean resistance sources. The objective of this work was to characterize 58 common bean cultivars with respect to their reaction to $P$. griseola races $31.17,63.19,63.23$ and 63.55 . The results showed that cultivars Antioquia 8 and CAL 143, both of Andean origin and Ecuador 299 and Mexico 235 (both of Mesoamerican origin) showed resistance to all four tested races. The cultivars A 193 and Golden Gate 416 showed resistance to three of the four tested races, and can be used as sources of resistance to angular leaf spot. All the other cultivars were susceptible to at least one of the four tested races. The most susceptible were: IPA 7419, Bambuí, CNC, Guanajuato 10-A-5, Black Diamond, Early Gallatin, Jamapa, Kentucky Wonder 780, and the "carioca" type cultivars AN 9022180, Aporé and Carioca 80. The new resistance sources identified in this work could be used in common bean breeding programs aiming to incorporate Andean and Mesoamerican resistance genes to angular leaf spot.
A mancha-angular do feijoeiro comum (Phaseolus vulgaris), causada pelo fungo Phaeoisariopsis griseola (Sacc.) Ferraris, encontra-se distribuída em todas as regiões do mundo onde essa leguminosa é cultivada. Segundo o Common wealth Mycological Institute esta enfermidade ocorre em mais de 60 países (Sartorato \& Rava, 1994). Estimativas de perdas na produção causadas pela mancha angular atingem a $70 \%$ no Brasil, dependendo das condições ambientais e de suscetibilidade dos cultivares (Rava et al., 1985; Sartorato \& Rava, 1992). As perdas são maiores quanto mais precoce for o aparecimento da doença.
A utilização de cultivares resistentes, é apresentada como a forma mais econômica de controle da doença (Sartorato et $a l .$, 1996). Entretanto, a alta variabilidade do patógeno torna difícil encontrar cultivares de feijoeiro com ampla e duradoura resistência a esta doença, sendo que ainda não foi identificado nenhum acesso de feijoeiro com resistência completa às mais virulentas raças de $P$. griseola (Guzmán et al., 1995, Nietsche et al., 2001). Em anos anteriores algumas fontes de resistência à mancha-angular já foram identificadas (Rava et al., 1985; Sartorato et al., 1991; Sartorato \& Rava, 1992; Faleiro et al., 2001). Entretanto, devido à grande variabilidade patogênica de 
Reação de cultivares de feijoeiro comum a quatro raças...

$P$. griseola, cultivares que se comportam como resistentes em determinadas regiões apresentam-se como suscetíveis em outras. Assim, estudos freqüentes da variabilidade genética do patógeno, bem como de sua distribuição geográfica e a determinação de fontes com adequado nível de resistência, são pontos-chave em programas de melhoramento visando resistência a esta doença.

Neste contexto, o objetivo do presente trabalho foi caracterizar 58 cultivares de feijoeiro, pertencentes ao banco de germoplasma do BIOAGRO/UFV, quanto à resistência à mancha-angular às raças $63.19,63.23,31.17$ e 63.55 de $P$. griseola previamente identificadas por Nietsche (2000).

O material genético utilizado foi cedido pela Embrapa Arroz e Feijão, Centro Internacional de Agricultura Tropical (CIAT), Universidade Federal de Lavras (UFLA), Departamento de Agricultura dos Estados Unidos (USDA) e Universidade de Michigan (EUA) (Tabela 1). Como testemunha resistente foi utilizada a cultivar México 54 (Niestche, 2000). Como testemunhas suscetíveis foram utilizadas as cultivares Rosinha G-2 e Rudá. Para a avaliação da resistência foram utilizadas 12 plantas por cultivar, sendo semeadas quatro sementes por vaso contendo $3 \mathrm{~kg}$ de solo, adubado com NPK, e mantidos em casa de vegetação.

As raças 63.19, 63.23, 31.17 e 63.55 de $P$. griseola utilizadas no presente trabalho pertencem a Micoteca do Programa de Melhoramento do Feijoeiro do BIOAGRO/UFV (Nietsche, 2000). Estas raças foram utilizadas em razão de sua elevada virulência e prevalência no Brasil, sendo obtidas de culturas monospóricas, mantidos em placa de petri ou tubo de ensaio contendo meio V8 (Campbell Soup Company, EUA). De cada tubo contendo a cultura do patógeno, foi obtida uma suspensão de conídios e fragmentos de micélio, a partir da adição de água estéril. Esta suspensão foi espalhada em placas contendo meio de suco de vegetais V8. Em seguida, as placas foram incubadas por 12 dias, no escuro, a 24?C. Este procedimento produziu altos níveis de esporulação.

A suspensão de conídios para inoculação foi obtida adicionando-se água destilada e raspando-se suavemente a superfície do meio com o auxílio de uma espátula, em seguida filtrada em gaze. Esta suspensão foi ajustada para a concentração final de $2 \times 10^{4}$ conídios $\mathrm{ml}^{-1}$, sendo feita a inoculação das plantas no estágio V3 (surgimento da primeira folha trifoliolada). A suspensão foi aplicada em ambas as faces das folhas, com auxílio de um atomizador De Vilbiss $n^{\circ} 15$, acionado por um compressor elétrico. As plantas foram, então, incubadas por um período de $48 \mathrm{~h}$ em câmara úmida e mantidas entre 20 e $22^{\circ} \mathrm{C}$ e $100 \%$ de umidade relativa, as quais foram, posteriormente, transferidas para casa de vegetação. Plantas inoculadas com diferentes raças foram mantidas em câmaras separadas.

A severidade da doença foi avaliada visualmente aos 15 e 21 dias após inoculação, usando-se a escala de nove graus descrita por Schoonhoven \& Pastor-Corrales (1987). Neste trabalho, as plantas com notas de 1 a 3 foram consideradas resistentes, de 4 a 6, com resistência intermediária e de 7 a 9 , suscetíveis.

Os resultados obtidos (Tabela 1) demonstram que as cultivares Antioquia 8 e CAL 143, de origem Andina, e Ecuador 299 e México 235, de origem Mesoamericana, foram resistentes às quatro raças utilizadas na inoculação. A cultivar Diacol Calima, de origem Andina apresentou reação de resistência a outras duas raças testadas e resistência intermediária às outras duas, podendo também ser uma fonte alternativa para programas de melhoramento visando resistência à mancha-angular. As cultivares A 193 e Golden Gate 416 mostraram resistência a três das quatro raças testadas. Resistência a duas das raças foi observada nas cultivares Costa Rica, Goytacazes, Manteigão Fosco 11, MDRK, México 279 e Novo Jalo. As cultivares AB 136, B98311, G 04658, ESAL 633, Kentucky Wonder 814, NEP 2 e Olathe, apresentaram resistência a uma das raças testadas. As cultivares AXS 37, California Small White 643, IAPAR 14 e Kaboon, além de possuírem resistência a uma raça, possuem, também, resistência intermediária a outras duas das raças utilizadas no trabalho. As cultivares AB 136, Catrachita, México 168, A 189 e G2333 apresentaram resistência a apenas uma das quatro raças testadas e resistência intermediária a uma das outras raças. As cultivares IPA 7419, Bambuí, Composto Negro Chimaltengo, Guanajuato 10-A-5, Diamante Negro, Early Gallatin, Jamapa e Kentucky Wonder 780 e as cultivares de sementes tipo “carioca” AN 9022180, Aporé e Carioca 80, foram suscetíveis a todas as raças testadas (Tabela 1).

Faleiro et al. (2001) já haviam observado a suscetibilidade das cultivares Carioca, Aporé, e Diamante Negro. A cultivar Novo Jalo, tida como suscetível por Faleiro et al. (2001) à raça 63.23 , foi avaliada como resistente no presente trabalho, provavelmente variações no experimento podem ter contribuído para as diferenças na manifestação da doença. Em outros trabalhos (Rava et al., 1985, Sartorato et al., 1996, Sartorato \& Rava, 1992), a cultivar Jalo EEP 558 é citada como resistente à mancha-angular. Entretanto, neste trabalho, esta cultivar foi suscetível às raças 63.19, 31.17 e 63.55 e apresentou resistência intermediária à raça 63.23, a mais prevalecente em Minas Gerais (Nietsche, 2000). A cultivar Caraota 260 também é mencionada como resistente à mancha-angular (Rava et al., 1985), porém neste trabalho, se comportou como suscetível a todas as raças testadas. Segundo Sartorato et al. (1991, 1996), as cultivares México 279, Costa Rica e Mineiro Precoce comportaram-se como resistentes ou moderadamente resistentes à enfermidade. Neste trabalho as cultivares México 279 e Costa Rica apresentaram resistência às raças 63.55 e 63.23, e a cultivar Mineiro Precoce apresentou resistência intermediária à raça 63.23 e suscetibilidade às outras raças testadas, o que confirma as observações de Sartorato et al. (1991, 1996). Em termos gerais, as discordâncias entre estes trabalhos podem ser devidas às diferenças de temperatura e umidade durante a inoculação, época de avaliação da resistência, número de plantas avaliadas, dentre outros fatores, que podem favorecer ou não o desenvolvimento e colonização pelo fungo e conseqüentemente influenciar na manifestação da doença.

Considerando que a maior parte do feijoeiro plantado no Brasil, pertence ao grupo mesoamericano, e que as raças de maior ocorrência são, por conseguinte, mesoamericanas (Nietsche et al., 2001), a piramidação de genes dos grupos 
E.J. Oliveira et al.

TABELA 1 - Características e reação das cultivares de feijoeiro (Phaseolus vulgaris) estudadas quanto a resistência às quatro raças de Phaeoisariopsis griseola

\begin{tabular}{|c|c|c|c|c|c|c|c|}
\hline \multirow{2}{*}{ Cultivar } & \multirow{2}{*}{ Origem } & \multirow{2}{*}{$\begin{array}{c}\text { Conjunto } \\
\text { Gênicd }\end{array}$} & \multirow{2}{*}{$\mathbf{T}^{2}$} & \multicolumn{4}{|c|}{ Raças } \\
\hline & & & & 63.19 & 63.55 & 63.23 & 31.17 \\
\hline A 189 & Colômbia & $\mathrm{A}$ & $\mathrm{G}$ & $\mathrm{S}$ & I & $\mathrm{R}$ & $\mathrm{S}$ \\
\hline A 193 & Colômbia & A & $\mathrm{G}$ & $\mathrm{R}$ & $\mathrm{S}$ & $\mathrm{R}$ & $\mathrm{R}$ \\
\hline $\mathrm{AB} 136$ & México & M & $\mathrm{M}$ & I & $\mathrm{S}$ & $\mathrm{S}$ & $\mathrm{R}^{3}$ \\
\hline AN 511608-0 & Brasil & M & $\mathrm{P}$ & $\mathrm{S}$ & I & $\mathrm{S}$ & $\mathrm{S}$ \\
\hline AN 9022180 & Brasil & $\mathbf{M}$ & $\mathrm{P}$ & $\mathrm{S}$ & $\mathrm{S}$ & $\mathrm{S}$ & $\mathrm{S}$ \\
\hline Antióquia 8 & Colômbia & A & $\mathrm{G}$ & $\mathrm{R}$ & $\mathrm{R}$ & $\mathrm{R}$ & $\mathrm{R}$ \\
\hline Aporé & Brasil & M & $\mathrm{P}$ & $\mathrm{S}$ & $\mathrm{S}$ & $\mathrm{S}$ & $\mathrm{S}$ \\
\hline Aurora & EUA & M & $\mathrm{P}$ & $\mathrm{I}^{3}$ & $S^{3}$ & $\mathrm{~S}$ & I \\
\hline AXS 37 & EUA & M & $\mathrm{P}$ & I & I & $\mathrm{S}$ & $\mathrm{R}$ \\
\hline В 98311 & EUA & $\mathbf{M}$ & $\mathrm{P}$ & $\mathrm{S}$ & $\mathrm{S}$ & $\mathrm{S}$ & $\mathrm{R}$ \\
\hline Bambuí & Brasil & M & $\mathrm{P}$ & $\mathrm{S}$ & $\mathrm{S}$ & $\mathrm{S}$ & $\mathrm{S}$ \\
\hline Brígida & Colômbia & M & $\mathrm{P}$ & $\mathrm{S}$ & I & $\mathrm{S}$ & $\mathrm{S}$ \\
\hline Brown Beauty & EUA & A & $\mathrm{M}$ & $\mathrm{S}$ & I & $\mathrm{S}$ & $\mathrm{S}$ \\
\hline CAL 143 & Colômbia & A & $\mathrm{G}$ & $\mathrm{R}$ & $\mathrm{R}$ & $\mathrm{R}$ & $\mathrm{R}$ \\
\hline California Small White 643 & EUA & M & $\mathrm{P}$ & I & I & $\mathrm{S}$ & $\mathrm{R}$ \\
\hline Caraota 260 & Venezuela & M & $\mathrm{P}$ & $\mathrm{S}$ & $\mathrm{S}$ & $\mathrm{S}$ & $\mathrm{S}$ \\
\hline Carioca & Brasil & M & $\mathrm{P}$ & I & $\mathrm{S}$ & $\mathrm{S}$ & $\mathrm{S}$ \\
\hline Carioca 80 & Brasil & M & $\mathrm{P}$ & $\mathrm{S}$ & $\mathrm{S}$ & $\mathrm{S}$ & $\mathrm{S}$ \\
\hline Catrachita & Honduras & M & $\mathrm{P}$ & $\mathrm{S}$ & $\mathrm{S}$ & $\mathrm{I}$ & $\mathrm{R}$ \\
\hline Compuesto Negro Chimaltengo & Guatemala & M & $\mathrm{P}$ & $\mathrm{S}$ & $\mathrm{S}$ & $\mathrm{S}$ & $\mathrm{S}$ \\
\hline Costa Rica & Costa Rica & M & $\mathrm{P}$ & $\mathrm{S}$ & $\mathrm{R}$ & $\mathrm{R}$ & $\mathrm{S}$ \\
\hline Diacol Calima & Colômbia & A & $\mathrm{G}$ & $\mathrm{I}$ & $\mathrm{R}$ & $\mathrm{R}$ & $\mathrm{I}$ \\
\hline Diamante Negro & Brasil & M & $\mathrm{P}$ & $\mathrm{S}$ & $\mathrm{S}$ & $\mathrm{S}$ & $\mathrm{S}$ \\
\hline Durango 222 & México & M & $\mathrm{M}$ & $\mathrm{S}$ & $\mathrm{S}$ & $\mathrm{S}$ & I \\
\hline Early Gallatin & EUA & $\mathrm{A} / \mathrm{M}$ & $\mathrm{M}$ & $\mathrm{S}$ & $\mathrm{S}$ & $\mathrm{S}$ & $\mathrm{S}$ \\
\hline Ecuador 299 & Equador & $\mathrm{M}$ & $\mathrm{M}$ & $\mathrm{R}$ & $\mathrm{R}$ & $\mathrm{R}$ & $\mathrm{R}$ \\
\hline Emgopa 201 Ouro & Colômbia & M & $\mathrm{P}$ & $\mathrm{S}$ & I & I & $\mathrm{S}$ \\
\hline G 04658 & Colômbia & M & $\mathrm{P}$ & $\mathrm{S}$ & $\mathrm{S}$ & $\mathrm{R}$ & $\mathrm{S}$ \\
\hline G 3817 & México & M & $\mathrm{P}$ & $\mathrm{S}$ & $\mathrm{S}$ & $\mathrm{S}$ & I \\
\hline Guanajuato $10-\mathrm{A}-5$ & Mexico & M & $\mathrm{P}$ & $\mathrm{S}$ & $\mathrm{S}$ & $\mathrm{S}$ & $\mathrm{S}$ \\
\hline IPA 7419 & Brasil & M & $P$ & $\mathrm{~S}$ & $\mathrm{~S}$ & $\mathrm{~S}$ & $\mathrm{~S}$ \\
\hline Mexico 168 & México & M & $\mathrm{M}$ & I & $\mathrm{S}$ & $\mathrm{S}$ & $\mathrm{R}$ \\
\hline Esal 633 & Brasil & M & $\mathrm{P}$ & $\mathrm{S}$ & $\mathrm{S}$ & $\mathrm{R}$ & $\mathrm{S}$ \\
\hline FT Bonito & Brasil & M & $\mathrm{P}$ & $\mathrm{S}$ & I & $\mathrm{S}$ & $\mathrm{S}$ \\
\hline G 2333 & México & M & $\mathrm{M}$ & $\mathrm{S}$ & I & $\mathrm{S}$ & $\mathrm{R}$ \\
\hline Golden Gate Wax & EUA & $\mathrm{A} / \mathrm{M}$ & $\mathrm{M}$ & $\mathrm{S}$ & $\mathrm{S}$ & $\mathrm{S}$ & I \\
\hline Golden Gate 416 & EUA & A? & $\mathrm{G}$ & $\mathrm{R}$ & $\mathrm{S}$ & $\mathrm{R}$ & $\mathrm{R}$ \\
\hline Goytacazes & Brasil & M & $\mathrm{P}$ & I & $\mathrm{R}$ & $\mathrm{R}$ & $\mathrm{S}$ \\
\hline IAPAR 14 & Brasil & M & $\mathrm{P}$ & $\mathrm{R}$ & I & $\mathrm{I}$ & $\mathrm{S}$ \\
\hline Ica Pijao & Colômbia & M & $\mathrm{P}$ & $\mathrm{S}$ & I & $\mathrm{S}$ & $\mathrm{S}$ \\
\hline Jalo EEP 558 & Brasil & $\mathrm{A}$ & $\mathrm{G}$ & $\mathrm{S}$ & $\mathrm{S}$ & $\mathrm{S}$ & I \\
\hline Jamapa & México & M & $\mathrm{P}$ & $\mathrm{S}$ & $\mathrm{S}$ & $\mathrm{S}$ & $\mathrm{S}$ \\
\hline Kaboon & Holanda & A & $\mathrm{G}$ & I & $\mathrm{S}$ & $\mathrm{I}$ & $\mathrm{R}$ \\
\hline Kentucky Wonder 765 & EUA & $\mathrm{A} / \mathrm{M}$ & $\mathrm{M}$ & I & $\mathrm{S}$ & $\mathrm{S}$ & $\mathrm{S}$ \\
\hline Kentucky Wonder 780 & EUA & $\mathrm{A} / \mathrm{M}$ & $\mathrm{M}$ & $\mathrm{S}$ & $\mathrm{S}$ & $\mathrm{S}$ & $\mathrm{S}$ \\
\hline Kentucky Wonder 814 & EUA & M & $\mathrm{M}$ & $\mathrm{S}$ & $\mathrm{S}$ & $\mathrm{S}$ & $\mathrm{R}$ \\
\hline Manteigao Fosco 11 & Brasil & M & $\mathrm{M}$ & $\mathrm{R}$ & I & $\mathrm{S}$ & $\mathrm{R}$ \\
\hline Michigan Dark Red Kidney & EUA & A & $\mathrm{G}$ & $\mathrm{S}$ & $\mathrm{S}$ & $\mathrm{R}$ & $\mathrm{R}$ \\
\hline Meia Noite & Brasil & $\mathbf{M}$ & $\mathrm{P}$ & $\mathrm{S}$ & $\mathrm{S}$ & I & $\mathrm{S}$ \\
\hline México 235 & México & M & $\mathrm{M}$ & $\mathrm{R}$ & $\mathrm{R}$ & $\mathrm{R}$ & $\mathrm{R}$ \\
\hline México 279 & México & M & $\mathrm{P}$ & $\mathrm{S}$ & $\mathrm{S}$ & $\mathrm{R}$ & $\mathrm{R}$ \\
\hline México 309 & México & $\mathrm{A} / \mathrm{M}$ & $\mathrm{M}$ & $\mathrm{S}$ & $\mathrm{S}$ & $\mathrm{S}$ & $\mathrm{S}$ \\
\hline Milionário & Colômbia & M & $\mathrm{P}$ & $\mathrm{S}$ & $\mathrm{S}$ & $\mathrm{I}$ & $\mathrm{S}$ \\
\hline Mineiro Precoce & Brasil & A & $\mathrm{G}$ & $\mathrm{S}$ & $\mathrm{S}$ & $\mathrm{S}$ & $\mathrm{I}$ \\
\hline N 98122 & - & M & $\mathrm{G}$ & $\mathrm{S}$ & $\mathrm{S}$ & $\mathrm{S}$ & $\mathrm{S}$ \\
\hline NEP 2 & Costa Rica & M & $\mathrm{P}$ & $\mathrm{S}$ & $\mathrm{S}$ & $\mathrm{R}$ & $\mathrm{S}$ \\
\hline Novo Jalo & Brasil & $\mathrm{A}$ & $\mathrm{G}$ & I & $\mathrm{S}$ & $\mathrm{R}$ & $\mathrm{R}$ \\
\hline Olathe & EUA & M & $\mathrm{M}$ & $\mathrm{S}$ & $\mathrm{S}$ & $\mathrm{S}$ & $\mathrm{R}$ \\
\hline México $54^{4}$ & & M & $\mathrm{M}$ & $\mathrm{R}$ & $\mathrm{R}$ & $\mathrm{R}$ & $\mathrm{R}$ \\
\hline Ruda $^{5}$ & Colômbia & M & $\mathrm{M}$ & $\mathrm{S}$ & $\mathrm{S}$ & $\mathrm{S}$ & $\mathrm{S}$ \\
\hline Rosinha G- $2^{5}$ & & $\mathrm{M}$ & $\mathrm{M}$ & $\mathrm{S}$ & $\mathrm{S}$ & $\mathrm{S}$ & $\mathrm{S}$ \\
\hline
\end{tabular}


Reação de cultivares de feijoeiro comum a quatro raças...

Andino e Mesoamericano seria uma estratégia eficiente na obtenção de resistência douradora à mancha-angular. Até o presente no Brasil, a única fonte supostamente Andina, de amplo espectro de resistência, é a cultivar AND 277 (Sartorato \& Rava, 1993). No entanto, os trabalhos de alelismo de Caixeta (2002) demonstraram que AND 277 possui três alelos de genes de resistência à mancha-angular provenientes de cultivares mesoamericanas, sugerindo que a resistência dessa cultivar tenha esta origem. A origem híbrida de AND 277 já foi sugerida anteriormente, devido à facilidade com que é cruzada com cultivares mesoamericanas (Voysest, 2000). Desta forma, as cultivares Andinas Antioquia 8 e CAL 143, podem ser fontes úteis de resistência em programas de melhoramento do feijoeiro no Brasil. Vieira et al. (2002), estudando o comportamento de cultivares de feijão do tipo manteigão em Minas Gerais, demonstraram que em condições de campo a cultivar CAL 143 foi resistente à ferrugem, oídio, mancha-angular e mancha-dealternária, e ainda foi tão produtiva quanto a cultivar Pérola (tipo carioca), com médias em nove ambientes, superior a 2700 $\mathrm{kg} / \mathrm{ha}$.

As cultivares Ecuador 299 e México 235 também podem ser de muita utilidade em programas de retrocruzamentos que utilizem cultivares mesoamericanas como genitores recorrentes, tendo em vista a relativa facilidade de transferência de genes entre indivíduos do mesmo conjunto gênico. Na genealogia da cultivar MAR-2, uma conhecida fonte de resistência à mancha angular, a cultivar Ecuador 299 aparece como genitor, indicando que esta possa ser a origem da resistência desta cultivar. Também, as cultivares Ecuador 299 e México 235 apresentam resistência a um grande número de raças de Uromyces appendiculatus (Pers) Unger, agente causador da ferrugem do feijoeiro (Pastor-Corrales, 2002). Com isso, existe a possibilidade de transferência concomitante de genes de resistência à mancha-angular e a ferrugem presentes nestas cultivares.

Os resultados deste trabalho são importantes para programas de melhoramento do feijoeiro no Brasil interessados na resistência à mancha angular. A busca de novas e melhores fontes de resistência tem sido uma constante preocupação, devido principalmente, à alta variabilidade patogênica do fungo $P$. griseola. Embora os resultados obtidos permitam considerar as cultivares Antioquia 8, CAL 143, Ecuador 299 e México 235 como boas fontes de resistência à manchaangular para serem utilizadas em programas de melhoramento, estudos adicionais devem ser realizados com o intuito de verificar a reação destas cultivares a outras raças do patógeno.

\section{REFERÊNCIAS BIBLIOGRÁFICAS}

CAIXETA, E.T. Caracterização da resistência genética à manchaangular e desenvolvimento de marcadores microssatélites para regiões específicas do genoma do feijoeiro. (Tese Doutorado) Genética e Melhoramento - Universidade Federal de Viçosa, 2002.

FALEIRO, F.G., NIETSCHE, S., RAGAGNIN, V.A., BORÉM, A., MOREIRA, M.A. \& BARROS, E.G. Resistência de cultivares de feijoeiro comum à ferrugem e a mancha-angular em condições de casa de vegetação. Fitopatologia Brasileira 26:86-89, 2001.

GUZMÁN, P., GILBERTSON, R. L., NODARI, R., JOHNSON, W.C., TEMPLE, S.R., MANDELA, D., MKANDAWIRE, A.B.C. \& GEPTS, P. Characterization of variability in the fungus Phaeoisariopsis griseola suggests coevolution with the common bean (Phaseolus vulgaris L.). Phytopathology 85:600-607. 1995.

NIETSCHE, S. Mancha-Angular do Feijoeiro-Comum: Variabilidade genética do patógeno e identificação de marcadores moleculares ligados à resistência. (Tese Doutorado) Genética e Melhoramento Universidade Federal de Viçosa, 2000.

NIETSCHE, S., BORÉM, A., CARVALHO, G.A., PAULA JR., T.J., FERREIRA, C.F., BARROS, E.G. \& MOREIRA, M.A. Genetic diversity of Phaeoisariopsis griseola in the state of Minas Gerais. Euphytica 117:77-84. 2001.

PASTOR-CORRALES, M.A. Apparent vulnerability of certain gene combinations for the management of Uromyces appendiculatus. Annual Report of the Bean Improvement Cooperative 45:40-41. 2002.

RAVA, S.C.A., SARTORATO, A. \& CARVALHO, J.R.P. Yield losses in dry bean (Phaseolus vulgaris L.) caused by angular leaf spot (Isariopsis griseola Sacc.). Annual Report of the Bean Improvement Cooperative 28:5-6. 1985.

SARTORATO, A., RAVA, C.A., MENTEN, J.O.M. \& BERGAMIMFILHO, A. Resistência vertical do feijoeiro comum (Phaseolus vulgaris L.) a Isariopsis griseola. Fitopatologia Brasileira 16:43-46. 1991.

SARTORATO, A. \& RAVA, C.A. Influência da cultivar e do número de inoculações na severidade da mancha-angular (Isariopsis griseola) e nas perdas na produção do feijoeiro comum (Phaseolus vulgaris). Fitopatologia Brasileira 17:247-251. 1992.

SARTORATO, A. \& RAVA, C.A. Determinação de resistência parcial do feijão comum a Isariopsis griseola. Resumo, Reunião Nacional de pesquisa de feijão, 1993, Londrina. 1993. Resumo 43.

SARTORATO, A. \& RAVA, C.A. Principais doenças do feijoeiro comum e seu controle. Brasília: EMBRAPA - SPI, 1994.

SARTORATO, A., RAVA, C.A. \& RIOS, G.P. Doenças fúngicas e bacterianas da parte aérea. In: Araújo, R.S., Rava, C.A., Stone, L.F. \& Zimmermann, M.J.O. (Eds.). Cultura do feijoeiro comum no Brasil. Piracicaba: POTAFÓS, 1996. pp.669-700.

SCHOONHOVEN, A. VAN \& PASTOR-CORRALES, MA. (Comps.). Standard system for evaluation of bean germplasm. Cali, Colombia: CIAT, 1987.

VIEIRA, R.F., VIEIRA, C., PINTO, C.M.F. \& RODRIGUES, O.L. Comportamento de cultivares de feijão do tipo manteigão em Minas Gerais - III. Revista Ceres 49:29-39. 2002.

VOYSEST, O.V. Mejoramiento genético del frijol (Phaseolus vulgaris L.): legado de variedades de América Latina 1930-1999. Cali, colombia, CIAT. 2000. 